\section{Research Square}

\title{
LINC00476 suppresses the progression of non- small cell lung cancer via inducing the ubiquitination of SETDB1
}

\section{Guanghui Cui}

Zhengzhou University First Affiliated Hospital

\section{Xiao Fu}

Zhengzhou University First Affiliated Hospital

\section{Wentao Wang}

Zhengzhou University First Affiliated Hospital

\section{Xiaofang Chen}

Zhengzhou University First Affiliated Hospital

\section{Shuaishuai Liu}

Zhengzhou University First Affiliated Hospital

\section{Peipei Cao}

Zhengzhou University First Affiliated Hospital

\section{Song Zhao ( $\backsim$ SongZhao8236@163.com )}

Zhengzhou University First Affiliated Hospital

\section{Guanghui Cui}

Zhengzhou University First Affiliated Hospital

\section{Research}

Keywords: LINC00476, non-small cell lung cancer, SETDB1, ubiquitination, protein stability

Posted Date: March 26th, 2020

DOI: https://doi.org/10.21203/rs.3.rs-19072/v1

License: (c) (1) This work is licensed under a Creative Commons Attribution 4.0 International License. Read Full License 


\section{Abstract}

Background: Long non-coding RNAs are involved in the tumorigenesis of non-small cell lung cancer (NSCLC). This study aims to investigate whether LINC00476 affects the proliferation, invasion, and migration of NSCLC cells via SETDB1-activated Wnt/ $\beta$-catenin pathway.

Method: The expression of LINC00476, SETDB1, Wnt1, and $\beta$-catenin were determined in NSCLC tumor tissues and the paired adjacent tissues, as well as in NSCLC cell lines and bronchial epithelioid cell lines. Cell proliferation, invasion, and migration were determined using cell counting kit-8 assay and Transwell assay. The relationship between LINC00476 and SETDB1 was elucidated using RNA pull-down, RNA immunoprecipitation, and ubiquitination assays.

Result: LINC00476 was significantly downregulated, while SETDB1, Wnt1, and $\beta$-catenin were upregulated in NSCLC tumor tissues and cell lines compared to the normal ones. Overexpression of LINC00476 promoted the proliferation, invasion, and migration of NSCLC cells, as well as suppressed tumor growth in the mouse xenograft. Meanwhile, overexpression of LINC00476 induced the degradation of SETDB1 via promoting its ubiquitination. The simultaneous overexpression of LINC00476 and SETDB1 negated the inhibition of LINC00476 overexpression on the proliferation, invasion, and migration of NSCLC cells.

Conclusion: LINC00476 acts as a tumor suppressor in NSCLC via downregulating SETDB1, which provides a novel target in treating NSCLC.

\section{Introduction}

In recent years, the high incidence of lung cancer has not only made it become one of the most common malignant tumors worldwide, but also been a major killer that threatens human health. Clinically, according to the pathological characteristics, lung cancers are divided into two types, the non-small cell lung cancer (NSCLC) which is about $80-85 \%$ of the total incidence of lung cancers, and the small cell lung cancer (SCLC) [1]. Although the diagnosis and treatment of NSCLC have made great progress from the tumor resection, chemotherapy, radiotherapy, targeted therapy, and even immune checkpoint blockade therapy [2], the non-specific symptoms in the early stage of NSCLC patients often result in a late stage and poor prognosis. Therefore, it is particularly important to clarify the molecular biological mechanisms of the development of NSCLC in order to elucidate novel therapeutic targets.

SET domain bifurcated 1 (SETDB1, also known as ESET or KMT1E) is a histone H3 lysine 9 methyltransferase [3], and it has been reported to be involved in the progression and aggressiveness of several kinds of cancers, including hepatocellular cancer [4], breast cancer [5], and colorectal cancer [6]. In NSCLC clinical samples and cell lines, SETDB1 was highly expressed, and the administration of SETDB1interfering drug mithramycin suppressed lung tumor growth in the nude mouse xenograft [7]. Meanwhile, the oncogenic effect of SETDB1 on NSCLC progression was demonstrated to be mediated via the Wnt/ $\beta$ catenin pathway $[8,9]$. However, the upstream molecular regulation of SETDB1 in NSCLS has not been fully elucidated. 
More than $90 \%$ of the genes in human genome do not encode proteins, and these genes are described as the non-coding RNAs (ncRNAs). Among them, the long non-coding RNAs (IncRNAs) are a subtype of ncRNAs, whose length is more than 200 nucleotides [10]. Initially, IncRNAs were considered as the "rubbish" of gene transcription. However, the increasing evidence has revealed the multiple functions of IncRNAs on cell fate regulation, especially on tumorigenesis [11]. For example, IncRNA-MALAT1 [12, 13] and IncRNA-XIST $[14,15]$ have been elucidated to regulate the proliferation, invasion, and migration of NSCLC. Still, there have been a large amount of IncRNAs whose effects on NSCLC tumorigenesis have not been clearly identified.

In the current study, we searched the GEO database and performed the statistical analysis to identify dysregulated IncRNAs in NSCLC tumorigenesis. We found that LINC00476 was markedly downregulated in NSCLC clinical tumor tissues and cell lines. Furthermore, our study demonstrated that LINC00476 modulated the proliferation, invasion, and migration of NSCLC cells and the tumor growth via affecting the ubiquitination of SETDB1, aiming to provide a novel therapeutic target in treating NSCLC.

\section{Materials And Methods}

\section{Clinical samples and cell lines}

NSCLC patients (50 cases) who were admitted to The First Affiliated Hospital of Zhengzhou University from January 2017 to January 2019 were included in this study. During the pulmonary tumor resection, fresh tumor tissues and the paired normal adjacent tissues were collected and were frozen at $-80{ }^{\circ} \mathrm{C}$.

Human bronchial epithelioid cell line (16HBE), human lung adenocarcinoma cell lines (SPC-A-1 and A549), human NSCLC cell line from a metastatic lymph node (H1299), human bronchioalveolar carcinoma cell line (H358), and human large cell lung cancer cell line (H460) were bought from Procell Life Technology (Wuhan, Hubei, China). They were cultured in the RPMI-1640 medium supplemented with $10 \%$ fetal bovine serum (FBS) and $1 \%$ penicillin/streptomycin at $37{ }^{\circ} \mathrm{C}\left(95 \%\right.$ air and $\left.5 \% \mathrm{CO}_{2}\right)$.

\section{Quantitative real-time PCR}

Quantitative real-time PCR (qRT-PCR) was used to determine the relative expressions of LINC00476 and the mRNA expression of SETDB1 in tissues and cells. Total RNAs were used for the reverse transcription reactions, and qRT-PCR was performed on a Step One Plus real-time system (Applied Biosystems, Foster City, CA, USA). GAPDH was used as an internal control. The relative expression was calculated using $2^{-}$ $\triangle \triangle \mathrm{Ct}$.

\section{Western blot analysis}

The protein expressions of SETDB1, Wnt1, $\beta$-catenin in tissues and cells were determined using western blot analysis. The primary antibodies used in this study were anti-SETDB1 (ab12317, 1:1000, Abcam, 
Cambridge, UK), anti-Wnt1 (ab15251, Abcam), anti- $\beta$-catenin (1:1000, \#8480, Cell Signaling Technology, Danvers, MA, USA), and anti- $\beta$-actin (ab8226, 1:500, Abcam, Cambridge, UK).

\section{Cell transfection}

The LINC00476-overexpressing plasmid (pcDNA-LINC00476) and its control plasmid (pcDNA), the small interfering RNA against LINC00476 (si-LINC00476) and its control RNA (si-control), the LINC00476overexpression lentivirus vector (Lenti-LINC00476) and its control lentivirus (Lenti-GFP), and the SETDB1overexpression lentivirus vector (Lenti-SETDB1) and its control lentivirus (Lenti-GFP) were obtained from RiboBio Technology (Guangzhou, Guangdong, China). Lipofectamine 2000 (Invitrogen, Waltham, MA, USA) was used to transfect the vectors into tumor cells in accordance with manufacture's constructions.

\section{Cell proliferation, invasion, and migration}

Cell proliferation of tumor cells was determined using Cell counting kit-8 (CCK-8) assay [16]. Tumor cells were seeded into 96 -well plates at a density of $5 \times 10^{4} / \mu \mathrm{L}$. The CCK-8 solution $(10 \mu \mathrm{L}$, Dojindo, Kumamoto, Japan) was added to each well for $0,24,48$, and $72 \mathrm{~h}$, respectively. The absorbance at $450 \mathrm{~nm}$ was measured by a microplate reader (Bio-Rad, Hercules, CA, USA).

Cell invasion and migration were determined using Transwell assay [17]. The pore size in the Transwell assay was $8.0 \mu \mathrm{m}$ (Costar, Shanghai, China). For cell migration assay, the upper chamber was coated with $200 \mu \mathrm{L}$ serum-free DMEM, while the lower chamber was coated with $600 \mu \mathrm{L}$ DMEM supplemented with $10 \%$ FBS. Tumor cells at a density of $1 \times 10^{5}$ per well were seeded into the upper chamber, followed by the culture for $24 \mathrm{~h}$. The cells in the lower chamber were fixed with methanol for $10 \mathrm{~min}$ and stained with crystal violet for 10 min. After being washed with PBS, cells were counted from five randomly selected fields at $200 \times$ magnification. For cell invasion assay, the membrane of the upper chamber was pre-coated with $30 \mathrm{mg} / \mathrm{cm}^{2}$ Matrigel (BD Biosciences, San Jose, CA, USA) for $1 \mathrm{~h}$. Then the performance was the same as the cell migration assay.

\section{In vivo experiments}

Male BALB/c nude mice $(n=10)$ were bought from the Shanghai Lab Animal Research Center (Shanghai, China). The xenograft model of lung cancer was established as previously described [18]. A549 cells were transiently transfected with Lenti-GFP/Lenti-LINC00476 for $48 \mathrm{~h}$. Then the cells were collected and were added to the mixture of FBS/Matrigel (1:1) before being injected into the posterior flank of the nude mouse ( $n=5$ in each group). The tumor volumes were measured every 3 days using a Vernier caliper (tumor volume $=$ length $\times$ width $^{2} \times 0.5$ ). Mice were sacrificed 21 days after the injection. The expressions of LINC00476 and SETDB1 in tumor tissues were determined using qRT-PCR and western blot analysis, respectively.

\section{RNA immunoprecipitation}


The interaction between LINC00479 and SETDB1 was determined in A549 cells using RNA immunoprecipitation (RIP). A Magna RIP RNA-Binding Protein Immunoprecipitation Kit (Millipore, Temecula, CA, USA) was used according to the manufacturer's instructions. SETDB1 antibody (ab12317, Abcam, Cambridge, UK) was used during the immunoprecipitation, and IgG was used as the control. The fold change of LINC00476 was determined using qRT-PCR.

\section{RNA pull-down assay}

The interaction between LINC00479 and SETDB1 was determined in A549 cells using RNA pull-down assay. Biotin-labeled LINC00479 and control probe (NC, negative control) were purchased from RiboBio Technology (Guangzhou, Guangdong, China). The probes were co-incubated with Streptavidin-labeled magnetic beads and $A 549$ cells overnight at $4^{\circ} \mathrm{C}$. Then the cells were lysed using RIPA buffer (Thermo Fisher Scientific, Waltham, MA, USA). After being incubated with magnetic beads for $1 \mathrm{~h}$, the cell lysates were collected and the protein expression of SETDB1 was detected using western blot analysis.

\section{Ubiquitination assay and Cycloheximide treatment}

A549 and H460 cells were transfected with HA-tagged ubiquitin (HA-Ub), Flag-tagged SETDB1 (FlagSETDB1), and pcDNA/pcDNA-LINC00476 for $48 \mathrm{~h}$, followed by the treatment of MG132 (a proteasome inhibitor). After MG132 treatment for $4 \mathrm{~h}$, cells were collected. The cell lysates were immunoprecipitated with SETDB1 antibody (ab12317, Abcam, Cambridge, UK) before being immunoblotted with HA antibody (ab9110, Abcam, Cambridge, UK). The protein expression of SETDB1 was determined using western blot analysis.

To investigate the effect of LINC00476 on the degradation of SETDB1, we transfected A549 and H460 cells with pcDNA/pcDNA-LINC00476, followed by the treatment of $125 \mu \mathrm{g} / \mathrm{mL}$ cycloheximide (CHX, a protein synthesis inhibitor). The protein expression of SETDB1 was determined at $0,1,2$, and $3 \mathrm{~h}$ after the $\mathrm{CHX}$ treatment using western blot analysis.

\section{Statistical analysis}

All data were presented as mean \pm standard deviation (SD). Data analysis imaging used SPSS 18.0 and Graphpad Prism 5.0. Statistical analyses between two groups were done by using the Student's t-test. One-way analysis of variance (ANOVA) followed by the LSD post hoc test was used to compare among multiple groups. Differences were considered statistically significant if $p<0.05$.

\section{Results}

\section{LINC00476 is lowly expressed in NSCLC tumor tissues and cell lines}

The GSE118370 dataset which refers to the invasive NSCLC samples in the GEO database (http://www.ncbi.nlm.nih.gov/geo/) showed that a large amount of IncRNAs and mRNAs were dysregulated in NSCLC as shown in the volcano plot (Fig. 1A). We screened top 15 downregulated 
IncRNAs and top 4 upregulated IncRNAs by adjusting p value $<0.05$ and logFC $<-1$ or $>1$ (Table. 1). Among them, LINC00476 was significantly downregulated in NSCLC tissues (adjust $p=0.027395$ ) by using GSE118370 dataset (Fig. 1B). Meanwhile, the overall survival (171 in high-level group and 430 in low-level group, $\mathrm{p}=0.0056, \mathrm{HR}=0.61,95 \% \mathrm{Cl}=0.42-0.87$ ) in NSCLC patients with low-level LINC00476 was shorter than those with high-level LINC00476 (Fig. 1C). These data suggested that the lower level of LINC00476 may be associated with poor prognosis of NSCLC patients. In the clinical tumor samples, which were collected during surgery by us, the relative LINC00476 expression was significantly lower than that in adjacent tissues $(0.362 \pm 0.144$ vs $0.998 \pm 0.157, p<0.01$, Fig. 1D). In addition, the low LINC00476 expression was related to the high grade of tumor stage and lymph node metastasis (both $p<0.01$, Table. 2). The protein expression of SETDB1 was upregulated in tumor tissues compared with that in adjacent tissues (Fig. 1E). The lowly expressed LINC00476 and the highly expressed SETDB1 were also determined in NSCLC cell lines, including SPC-A-1, A549, H1299, H460, and H358, in comparison with normal bronchial epithelioid cell line (16HBE) (Fig. 1F). Moreover, the downregulation of LINC00476 in A549 and H460 cells were more obvious than other NSCLC cell lines $(p<0.01$ vs $p<0.05)$. The protein expressions of Wnt1 and $\beta$-catenin were upregulated in NSCLC cell lines compared to the normal bronchial epithelioid cell line (Fig. 1F).

\section{Overexpression of LINC00476 inhibits NSCLC cell proliferation, migration, and invasion in vitro and suppresses NSCLC tumor growth in vivo}

Given that LINC00476 expression was dramatically reduced in A549 and H460 cells, we used these cells in the next experiments. After overexpressing LINC00476 via pcDNA-LINC00476 transfection, the relative expression of LINC00476 was markedly induced ( $p<0.01$, Fig. 2A). The overexpression of LINC00476 significantly suppressed cell proliferation of A549 cells at $24 \mathrm{~h}(0.240 \pm 0.036$ vs $0.467 \pm 0.033, p=$ $0.040), 48 \mathrm{~h}(0.400 \pm 0.062$ vs $0.660 \pm 0.029, p=0.037)$, and $72 \mathrm{~h}(0.517 \pm 0.057$ vs $1.080 \pm 0.086, p=$ $0.009)$ and $\mathrm{H} 460$ cells at $24 \mathrm{~h}(0.297 \pm 0.034$ vs $0.597 \pm 0.068, p=0.007), 48 \mathrm{~h}(0.383 \pm 0.040$ vs $0.677 \pm$ $0.050, p=0.044)$, and $72 \mathrm{~h}(0.503 \pm 0.040$ vs $1.220 \pm 0.106, p=0.019)$ (Fig. 2B). The overexpression of LINC00476 also inhibited cell invasion of H460 cells (91.000 \pm 18.493 vs $263.000 \pm 18.019, p=0.022$ ) and A549 cells (192.333 \pm 16.214 vs $408.667 \pm 20.039, p=0.008)$ (Fig. 2 C), as well as cell migration of H460 cells ( $397.333 \pm 20.270$ vs $616.667 \pm 28.674, p=0.021)$ and A549 cells (366.000 \pm 22.760 vs $669.333 \pm 25.091, p=0.000$ ) (Fig. 2D). For the in vivo experiments, the overexpression of LINC00476 markedly suppressed the tumor growth during the 21 days (Fig. 2E). Then the upregulated expression of LINC00476 (Fig. 2F) and the downregulated expressions of SETDB1, Wnt1, and $\beta$-catenin (Fig. 2G) were confirmed in tumor tissues of Lenti-LINC00476 injected mice compared with the Lenti-GFP injected mice.

\section{LINC00476 induces the degradation of SETDB1 via promoting its ubiquitination}

SETDB1 was expressed in the LINC00476 pull-down compound (Fig. 3A), and the fold change of LINC00476 was increased in SETDB1-immunoprecipitated complex compared with the lgGimmunoprecipitated complex ( $p<0.05$, Fig. 3B), suggesting the interaction between LINC00476 and SETDB1. In A549 and H460 cells, the overexpression of LINC00476 reduced SETDB1 protein expression, 
while the knockdown of LINC00476 enhanced SETDB1 protein expression (Fig. 3C), indicating the negative regulatory relationship between LINC00476 and SETDB1. However, either overexpressing or knocking down LINC00476 did not affect the mRNA level of SETDB1 ( $p>0.05$, Fig. 3D). Therefore, we supposed that LINC00476 modulated the protein stability of SETDB1. After overexpressing LINC00476 in A549 and H460 cells, the degradation of SETDB1 protein was accelerated at 1, 2, and $3 \mathrm{~h}$ after the treatment of the protein synthesis inhibitor CHX (Fig. 3E). In addition, the overexpression of LINC00476 increased the ubiquitin-bound SETDB1 (Fig. 3F), suggesting that LNC00476 induced the degradation of SETDB1 protein via increasing its ubiquitination.

\section{LINC00476 suppresses NSCLC cell proliferation, invasion, and migration via the SETDB1/Wnt/ $\beta$-catenin axis}

A549 and H460 cells were transfected with LINC00476 overexpression plasmid (pcDNA-LINC00476), or co-transfected with SETDB1 overexpression lentivirus (Lenti-SETDB1) for $48 \mathrm{~h}$. The overexpression of LINC00476 significantly inhibited the cell proliferation, while such response was negated by the simultaneous overexpression of LINC00476 and SETDB1 (Fig. 4A). Similarly, the co-transfection restored the inhibition of cell invasion and migration which were caused by LINC00746 overexpression (Fig. 4B \& 4C). These data indicated that LINC00476 suppressed the cell proliferation, invasion, and migration of NSCLC cells via modulating SETDB1. In addition, the co-transfection restored the reduction of SETDB1, Wnt1, and $\beta$-catenin protein levels which were downregulated by LINC00476 overexpression (Fig. 4D). Taken together, our findings indicated that LINC00476 suppressed NSCLC cell proliferation, invasion, and migration via the SETDB1/Wnt/ $\beta$-catenin axis.

\section{Discussion}

The critical regulatory functions of IncRNAs have been elucidated recently, especially in cancers [19]. In the current study, a novel functional InCRNA, LINC00476, was identified in NSCLC, and it acted as a tumor suppressor via inducing the ubiquitination and degradation of the oncogenic protein SETDB1. Our findings provide a new insight into the mechanism of LINC00476/SETDB1 axis in NSCLC, suggesting that LINC00476 may be a potential therapeutic target of NSCLC.

LncRNAs have been shown to be implicated in multiple biological functions of cells, including cell cycle progression, cell differentiation, gene expression regulation, and chromatin modification [20]. In NSCLC, these functions of IncRNAs make them to be valuable in cancer diagnosis, treatment, and predicting and improving prognosis. A study conducted by Weber et al showed that the expression of serum IncRNAMALAT1 was dysregulated in 45 NSCLC patients in comparison with healthy controls, indicating IncRNAMALAT1 as a promising diagnostic biomarker due to its high stability, specificity, and minimal invasiveness [21]. LncRNA-HOTAIR was demonstrated to contribute to cisplatin resistance in lung adenocarcinoma cells via the downregulation of p21 expression [22], thus the knockdown of IncRNAHOTAIR by RNA interference technologies has been shown to reduce viability and invasiveness of lung 
cancer [23]. The elevation of IncRNA-H19 predicted a poor prognosis of NSCLC patients and was an independent prognostic factor for overall survival [24]. Inspired by these studies, we searched the GEO database for the potential dysregulated IncRNAs in NSCLC samples, and we found that LINC00476 was significantly downregulated in NSCLC tumor tissues in comparison with the paired normal tissues. Consistent with the dataset, the downregulated LINC00476 was also determined in clinical tumor samples compared with the adjacent tissues. In addition, the low expression of LINC00476 was relative to the high clinical stage and lymph node metastasis, indicating LINC00476 may be a protective factor against NSCLC.

LINC00476 is a newly found IncRNA whose functions have not been fully understood. LINC00476 is located in chromosome 9 at q22.32, and is expressed in many types of tissues, including in the lungs. In our study, the expression of LINC00476 was relatively lower in NSCLC tumor tissues than that in the paired adjacent tissues. Similarly, the expression of LINC00476 was relatively lower in five NSCLC cell lines (SPC-A-1, A549, H1299, H460, and H358) than that in the bronchial epithelioid cell line (16HBE). Moreover, the downregulation of LINC00476 in A549 and H460 cell lines seemed more obvious than in SPC-A-1, H1299, and H358 cell lines ( $p<0.01$ vs $p<0.05)$. Since these cell lines were originated from different types of NSCLC, such as adenocarcinoma and large cell carcinoma, whether the expression of LINC00476 is associated with lung cancer cell types deserves further investigations.

The regulatory functions of IncRNAs can be exerted by several ways. The IncRNA-protein interaction plays an important role in the posttranslational modification of proteins via affecting the protein stability [25]. For instance, IncRNA-XLOC_006390 was reported to promote pancreatic carcinogenesis via blocking c-Myc ubiquitination and stabilizing c-Myc protein [26]. In the current study, the binding between LINC00476 and SETDB1 was confirmed using RNA pull-down and RIP assays. The unchanged mRNA level while the negatively changed protein level of SETDB1 by overexpressing or knocking down LINC00476 suggested that the SETDB1 expression may be regulated by LINC00476 at posttranslational levels. The further CHX experiment and ubiquitination assay indicated that the LINC00476-SETDB1 interaction was modulated by LINC00476-induced ubiquitination and degradation of SETDB1. Our findings provide more evidence in IncRNA-protein interactions in NSCLC.

In conclusion, this study indicated that LINC00476 acted as a tumor suppressor in NSCLC. The overexpression of LINC00476 may be a potential therapeutic strategy in treating NSCLC.

\section{Declarations}

\section{Ethics approval and consent to participate}

This study was approved by the Institute Research Medical Ethics Committee of The First Affiliated Hospital of Zhengzhou University. Written informed consents were obtained from all the patients involved.

\section{Funding}


None.

\section{Availability of data and material}

The datasets used and/or analyzed during the current study are available from the corresponding author on reasonable request.

\section{Competing interests}

All authors declare that they have no competing interests.

\section{References}

[1] J.A. Howington, M.G. Blum, A.C. Chang, A.A. Balekian, S.C. Murthy, Treatment of stage I and II nonsmall cell lung cancer: Diagnosis and management of lung cancer, 3rd ed: American College of Chest Physicians evidence-based clinical practice guidelines, Chest, 143 (2013) e278S-e313S.

[2] W. Jing, M. Li, Y. Zhang, F. Teng, A. Han, L. Kong, H. Zhu, PD-1/PD-L1 blockades in non-small-cell lung cancer therapy, OncoTargets and therapy, 9 (2016) 489-502.

[3] D.C. Schultz, K. Ayyanathan, D. Negorev, G.G. Maul, F.J. Rauscher, 3rd, SETDB1: a novel KAP-1associated histone $\mathrm{H} 3$, lysine 9-specific methyltransferase that contributes to HP1-mediated silencing of euchromatic genes by KRAB zinc-finger proteins, Genes Dev, 16 (2002) 919-932.

[4] C.M. Wong, L. Wei, C.T. Law, D.W. Ho, F.H. Tsang, S.L. Au, K.M. Sze, J.M. Lee, C.C. Wong, I.O. Ng, Upregulation of histone methyltransferase SETDB1 by multiple mechanisms in hepatocellular carcinoma promotes cancer metastasis, Hepatology, 63 (2016) 474-487.

[5] J. Batham, P.S. Lim, S. Rao, SETDB-1: A Potential Epigenetic Regulator in Breast Cancer Metastasis, Cancers (Basel), 11 (2019).

[6] K. Chen, F. Zhang, J. Ding, Y. Liang, Z. Zhan, Y. Zhan, L.H. Chen, Y. Ding, Histone Methyltransferase SETDB1 Promotes the Progression of Colorectal Cancer by Inhibiting the Expression of TP53, J Cancer, 8 (2017) 3318-3330.

[7] M. Rodriguez-Paredes, A. Martinez de Paz, L. Simo-Riudalbas, S. Sayols, C. Moutinho, S. Moran, A. Villanueva, M. Vazquez-Cedeira, P.A. Lazo, F. Carneiro, C.S. Moura, J. Vieira, M.R. Teixeira, M. Esteller, Gene amplification of the histone methyltransferase SETDB1 contributes to human lung tumorigenesis, Oncogene, 33 (2014) 2807-2813.

[8] Q.Y. Sun, L.W. Ding, J.F. Xiao, W. Chien, S.L. Lim, N. Hattori, L. Goodglick, D. Chia, V. Mah, M. Alavi, S.R. Kim, N.B. Doan, J.W. Said, X.Y. Loh, L. Xu, L.Z. Liu, H. Yang, T. Hayano, S. Shi, D. Xie, D.C. Lin, H.P. Koeffler, SETDB1 accelerates tumourigenesis by regulating the WNT signalling pathway, J Pathol, 235 (2015) 559570. 
[9] M. Shapiro, G. Akiri, C. Chin, J.P. Wisnivesky, M.B. Beasley, T.S. Weiser, S.J. Swanson, S.A. Aaronson, Wnt pathway activation predicts increased risk of tumor recurrence in patients with stage I nonsmall cell lung cancer, Annals of surgery, 257 (2013) 548-554.

[10] X. Sun, L.-h. Luo, K.-z. Zhong, J.-r. Li, LncRNA PCAT29 Suppresses Cell Proliferation, Invasion, and Migration in Renal Carcinoma by Regulating FLOT1, Clinical Surgery Research Communications, 2 (2018) 35-42.

[11] C. Lin, L. Yang, Long Noncoding RNA in Cancer: Wiring Signaling Circuitry, Trends in cell biology, 28 (2018) 287-301.

[12] L.H. Schmidt, T. Spieker, S. Koschmieder, S. Schaffers, J. Humberg, D. Jungen, E. Bulk, A. Hascher, D. Wittmer, A. Marra, L. Hillejan, K. Wiebe, W.E. Berdel, R. Wiewrodt, C. Muller-Tidow, The long noncoding MALAT-1 RNA indicates a poor prognosis in non-small cell lung cancer and induces migration and tumor growth, Journal of thoracic oncology : official publication of the International Association for the Study of Lung Cancer, 6 (2011) 1984-1992.

[13] L.H. Schmidt, D. Gorlich, T. Spieker, C. Rohde, M. Schuler, M. Mohr, J. Humberg, T. Sauer, N.H. Thoenissen, A. Huge, R. Voss, A. Marra, A. Faldum, C. Muller-Tidow, W.E. Berdel, R. Wiewrodt, Prognostic impact of Bcl-2 depends on tumor histology and expression of MALAT-1 IncRNA in non-small-cell lung cancer, Journal of thoracic oncology : official publication of the International Association for the Study of Lung Cancer, 9 (2014) 1294-1304.

[14] J. Liu, L. Yao, M. Zhang, J. Jiang, M. Yang, Y. Wang, Downregulation of LncRNA-XIST inhibited development of non-small cell lung cancer by activating miR-335/SOD2/ROS signal pathway mediated pyroptotic cell death, Aging, 11 (2019) 7830-7846.

[15] J. Wang, H. Cai, Z. Dai, G. Wang, Down-regulation of IncRNA XIST inhibits cell proliferation via regulating miR-744/RING1 axis in non-small cell lung cancer, Clinical science (London, England : 1979), 133 (2019) 1567-1579.

[16] X. Kang, F. Kong, K. Huang, L. Li, Z. Li, X. Wang, W. Zhang, X. Wu, LncRNA MIR210HG promotes proliferation and invasion of non-small cell lung cancer by upregulating methylation of CACNA2D2 promoter via binding to DNMT1, OncoTargets and therapy, 12 (2019) 3779-3790.

[17] X. Kong, Y. Zhao, X. Li, Z. Tao, M. Hou, H. Ma, Overexpression of HIF-2alpha-Dependent NEAT1 Promotes the Progression of Non-Small Cell Lung Cancer through miR-101-3p/SOX9/Wnt/beta-Catenin Signal Pathway, Cellular physiology and biochemistry : international journal of experimental cellular physiology, biochemistry, and pharmacology, 52 (2019) 368-381.

[18] J. Lv, M. Qiu, W. Xia, C. Liu, Y. Xu, J. Wang, X. Leng, S. Huang, R. Zhu, M. Zhao, F. Ji, L. Xu, K. Xu, R. Yin, High expression of long non-coding RNA SBF2-AS1 promotes proliferation in non-small cell lung cancer, Journal of experimental \& clinical cancer research : CR, 35 (2016) 75. 
[19] A.M. Schmitt, H.Y. Chang, Long Noncoding RNAs in Cancer Pathways, Cancer cell, 29 (2016) 452-463.

[20] J. Beermann, M.T. Piccoli, J. Viereck, T. Thum, Non-coding RNAs in Development and Disease: Background, Mechanisms, and Therapeutic Approaches, Physiological reviews, 96 (2016) 1297-1325.

[21] D.G. Weber, G. Johnen, S. Casjens, O. Bryk, B. Pesch, K.H. Jockel, J. Kollmeier, T. Bruning, Evaluation of long noncoding RNA MALAT1 as a candidate blood-based biomarker for the diagnosis of non-small cell lung cancer, BMC research notes, 6 (2013) 518.

[22] Z. Liu, M. Sun, K. Lu, J. Liu, M. Zhang, W. Wu, W. De, Z. Wang, R. Wang, The long noncoding RNA HOTAIR contributes to cisplatin resistance of human lung adenocarcinoma cells via downregualtion of p21(WAF1/CIP1) expression, PLoS One, 8 (2013) e77293.

[23] Y. Yao, J. Li, L. Wang, Large intervening non-coding RNA HOTAIR is an indicator of poor prognosis and a therapeutic target in human cancers, Int J Mol Sci, 15 (2014) 18985-18999.

[24] E. Zhang, W. Li, D. Yin, W. De, L. Zhu, S. Sun, L. Han, c-Myc-regulated long non-coding RNA H19 indicates a poor prognosis and affects cell proliferation in non-small-cell lung cancer, Tumour Biol, 37 (2016) 4007-4015.

[25] J.R. Alvarez-Dominguez, H.F. Lodish, Emerging mechanisms of long noncoding RNA function during normal and malignant hematopoiesis, Blood, 130 (2017) 1965-1975.

[26] J. He, F. Li, Y. Zhou, X. Hou, S. Liu, X. Li, Y. Zhang, X. Jing, L. Yang, LncRNA XLOC_006390 promotes pancreatic carcinogenesis and glutamate metabolism by stabilizing c-Myc, Cancer Lett, (2019).

\section{Tables}

Table 1. Downregulated and upregulated IncRNAs in tumor tissues of NSCLC patients 


\begin{tabular}{|lllll|}
\hline Gene symbol & ID & Adjust P value & P value & logFC \\
\hline Downregulated IncRNAs & & & & \\
\hline LOC101930541 & 1561211_at & 0.002532 & $9.99 \mathrm{e}-07$ & -2.6812646 \\
\hline PCAT19 & 230132_at & 0.002997 & $2.14 \mathrm{e}-06$ & -2.2812843 \\
\hline LOC101927699 & 244308_at & 0.005738 & $1.27 \mathrm{e}-05$ & -2.5506702 \\
\hline LINC00312 & 220244_at & 0.007418 & $2.63 \mathrm{e}-05$ & -2.3581367 \\
\hline LOC101060604 & 1555216_a_at & 0.009194 & $4.21 \mathrm{e}-05$ & -1.7056772 \\
\hline LOC101928612 & 232227_at & 0.009813 & $4.91 \mathrm{e}-05$ & -2.0472014 \\
\hline LOC101928551 & 221337_s_at & 0.01742 & $1.52 \mathrm{e}-04$ & -1.8459938 \\
\hline LOC101927069 & 1560169_at & 0.017712 & $1.59 \mathrm{e}-04$ & -1.9597048 \\
\hline LINC00312 & 240306_at & 0.018904 & $1.83 \mathrm{e}-04$ & -2.7696985 \\
\hline LINC01359 & 1562103_at & 0.020012 & $2.07 \mathrm{e}-04$ & -1.9505817 \\
\hline LINC00845 & 238298_at & 0.025347 & $3.44 \mathrm{e}-04$ & -2.6691229 \\
\hline CARMN & 1558828_s_at & 0.026325 & $3.71 \mathrm{e}-04$ & -1.775856 \\
\hline LINC00476 & 239799_at & $\mathbf{0 . 0 2 7 3 9 5}$ & $4.06 \mathrm{e}-04$ & -1.5113358 \\
\hline LOC101929398 & 1560826_at & 0.02797 & $4.33 \mathrm{e}-04$ & -2.997071 \\
\hline PTCSC1 & 233111_at & 0.030061 & $4.88 \mathrm{e}-04$ & -1.1507019 \\
\hline Upregulated IncRNAs & & & & \\
\hline BLACAT1 & 232105_at & 0.003996 & $5.85 \mathrm{e}-06$ & 2.224308 \\
\hline LINC01123/LINC01106 & 242222_at & 0.009194 & $3.97 \mathrm{e}-05$ & 2.2867043 \\
\hline LOC105369635 & 240284_x_at & 0.025186 & $3.34 \mathrm{e}-04$ & 1.2656658 \\
\hline LOC100505938 & 230641_at & 0.030278 & $5.06 \mathrm{e}-04$ & 1.5238666 \\
\hline
\end{tabular}

NSCLC, non-small cell lung cancer. FC, fold change.

Table 2. The association of LINC00476 expression with clinicopathological features of NSCLC patients 


\begin{tabular}{|c|c|c|c|c|}
\hline \multirow[t]{2}{*}{ Clinicopathological features } & \multirow{2}{*}{$\begin{array}{l}\text { Sample } \\
(n=50)\end{array}$} & \multicolumn{2}{|c|}{ LINC00476 expression } & \multirow{2}{*}{$\begin{array}{l}\text { P value } \\
\text { (High vs Low) }\end{array}$} \\
\hline & & Low $(n=28)$ & High $(n=22)$ & \\
\hline Gender & & & & 0.826 \\
\hline Male & 23 & 13 & 10 & \\
\hline Female & 27 & 15 & 12 & \\
\hline Age (years) & & & & 0.613 \\
\hline$<65$ & 29 & 16 & 13 & \\
\hline$\geq 65$ & 21 & 12 & 9 & \\
\hline Smoking history & & & & 0.545 \\
\hline Yes & 26 & 14 & 12 & \\
\hline No & 24 & 14 & 10 & \\
\hline Histological subtype & & & & 0.437 \\
\hline Squamous cell carcinoma & 22 & 15 & 7 & \\
\hline Adenocarcinoma & 28 & 13 & 15 & \\
\hline Tumor size $(\mathrm{cm})$ & & & & 0.329 \\
\hline$\leq 5$ & 24 & 15 & 9 & \\
\hline$>5$ & 26 & 11 & 15 & \\
\hline TNM stage & & & & 0.003 \\
\hline$|-I|$ & 24 & 6 & 18 & \\
\hline III-IV & 26 & 22 & 4 & \\
\hline Lymph node metastasis & & & & 0.001 \\
\hline Yes & 30 & 21 & 9 & \\
\hline No & 20 & 7 & 13 & \\
\hline
\end{tabular}

NSCLC, non-small cell lung cancer.

Figures 
A

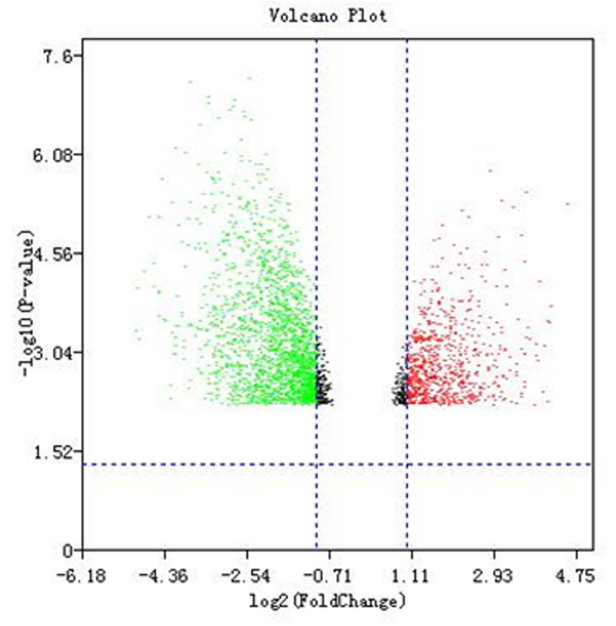

B

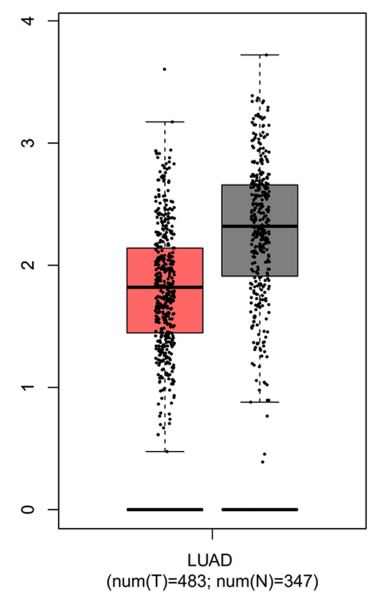

C

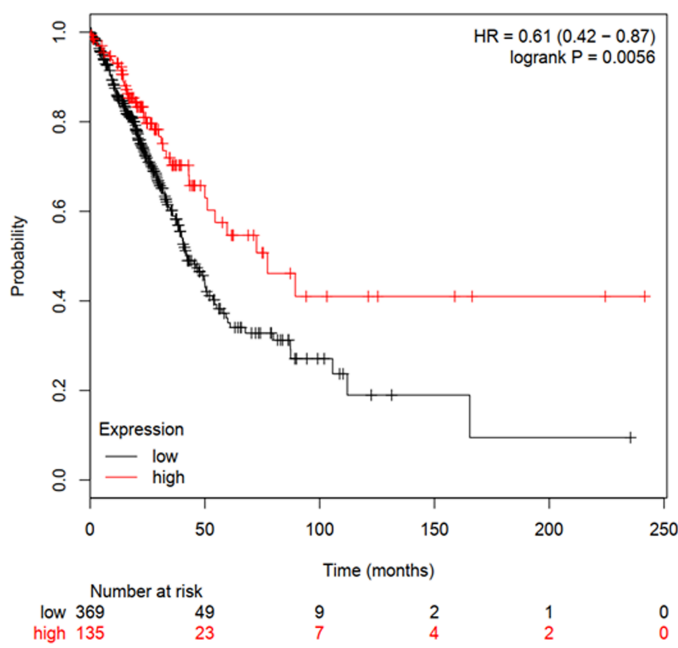

D

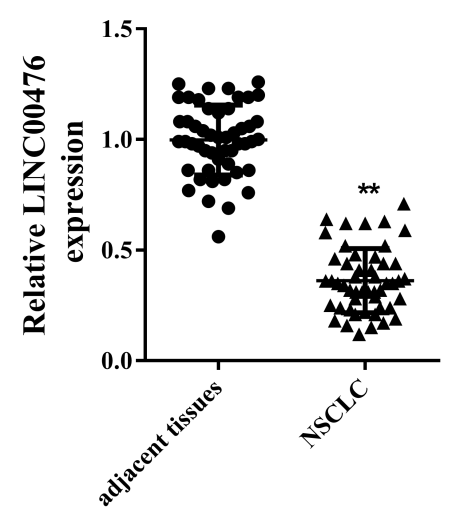

$\mathrm{E}$

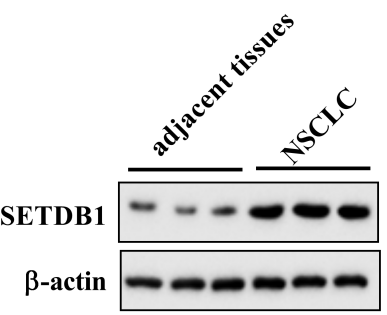

$\mathbf{F}$
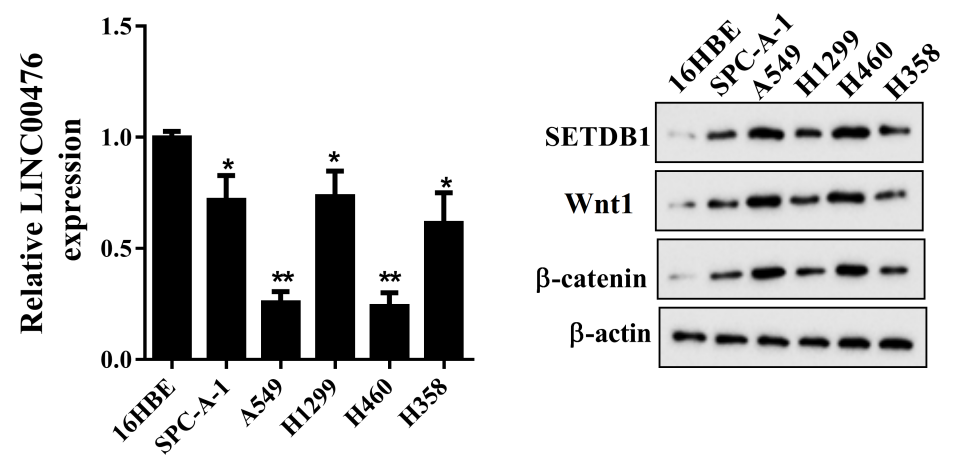

Figure 1

LINC00476 is lowly expressed in NSCLC tumor tissues and cell lines A. The volcano plot of dysregulated IncRNAs and mRNAs in NSCLC samples (GSE118370 dataset) by searching the GEO database. B. The $\log$ FC of LINC00476 was analyzed by using GSE118370 dataset. C. The overall survival in NSCLC patients with different levels of LINC00476 expression. D. The relative expression of LINC00476 in NSCLC tumor tissues and adjacent tissues was determined using qRT-PCR. E. The protein expression of SETDB1 in NSCLC tumor tissues and adjacent tissues was determined using western blot analysis. F. The relative expression of LINC00476 and the protein expressions of SETDB1, Wnt1, and $\beta$-catenin in normal bronchial epithelioid cell line (16HBE) and in NSCLC cell lines (SPC-A-1, A549, H1299, H460, and H358) were determined using qRT-PCR and western blot analysis, respectively. ${ }^{\star} p<0.05,{ }^{\star *} p<0.01$ vs adjacent tissues or $16 \mathrm{HBE}$. 
A

B

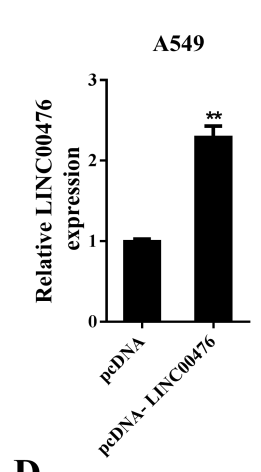

D
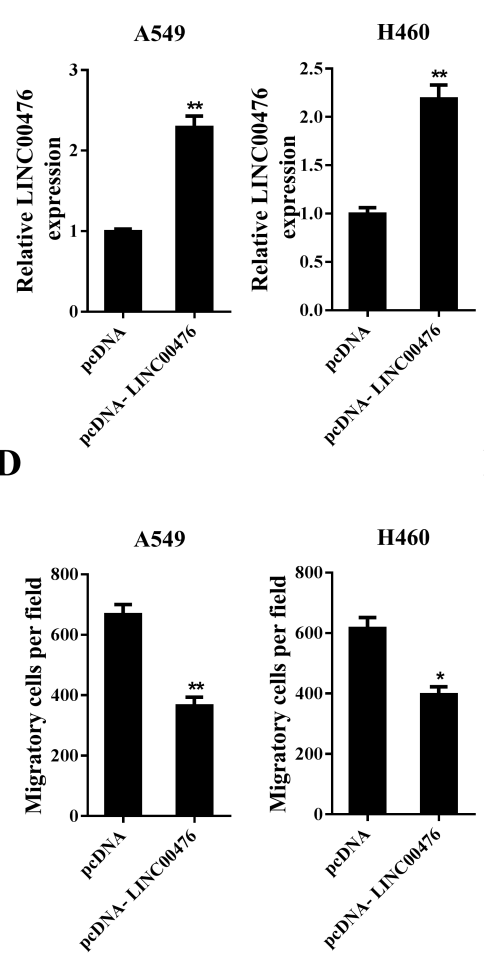

$\mathbf{E}$

A549
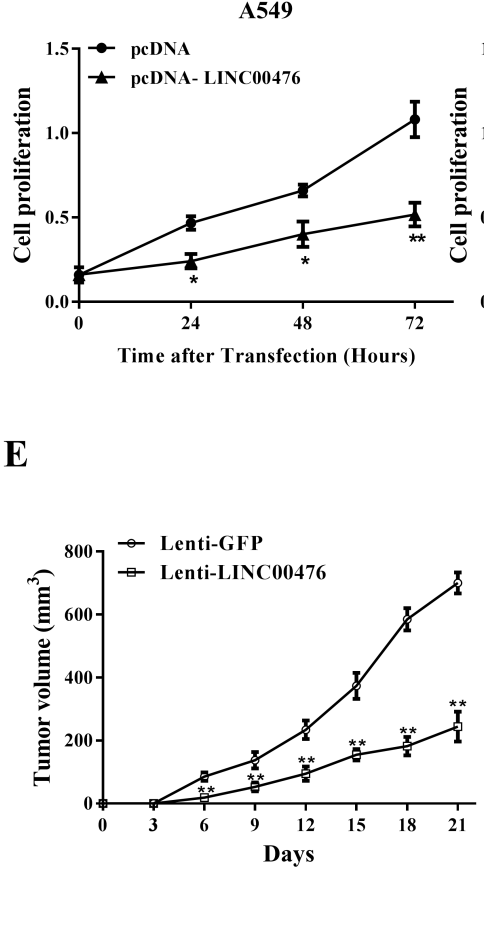

C
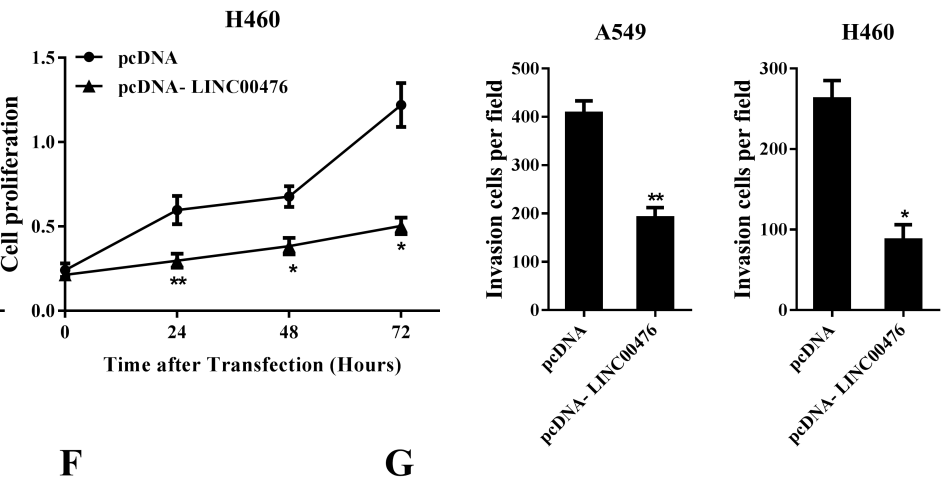

G

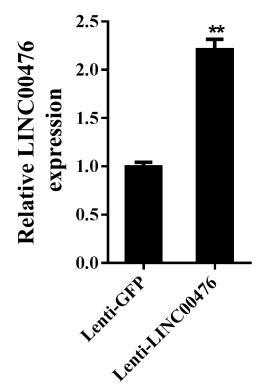

Figure 2

Overexpression of LINC00476 inhibits NSCLC cell proliferation, migration, and invasion in vitro and suppresses NSCLC tumor growth in vivo NSCLC cell lines A549 and H460 cells were transfected with LINC00476 overexpression plasmid (pcDNA-LINC00476) or the control plasmid (pcDNA) for $48 \mathrm{~h}$. The relative expression of LINC00476 (A), the cell proliferation (B), the cell invasion (C) and the cell migration (D) were determined using qRT-PCR, cell counting kit-8 (CCK-8) assay, and Transwell assay. A549 cells were transfected with LINC00476 overexpression lentivirus (Lenti-LINC00476) or the control lentivirus (Lenti-GFP) for $48 \mathrm{~h}$. BALB/c nude mice were injected with the transfected A549 cells which were mixed with FBS/Matrigel (1:1) solution in the posterior flank ( $n=5$ in each group). E. The tumor volumes were measured every 3 days using vernier caliper (tumor volume= length $\times$ width $2 \times 0.5$ ). The relative expression of LINC00476 (F) and the protein expressions of SETDB1, Wnt1, and $\beta$-catenin (G) were determined in tumor tissues using qRT-PCR and western blot analysis. ${ }^{\star} p<0.05,{ }^{\star *} p<0.01$ vs pcDNA or Lenti-GFP. 
A

B

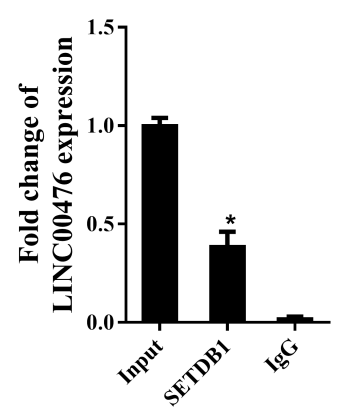

C

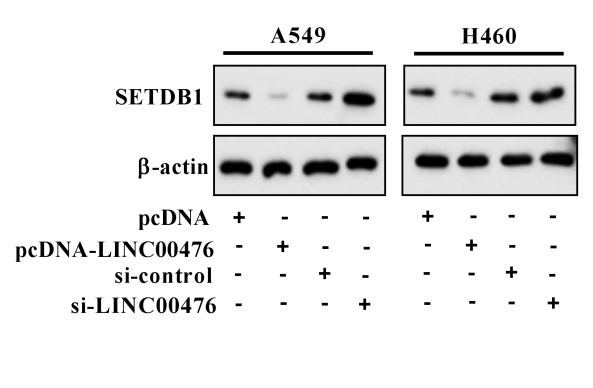

F

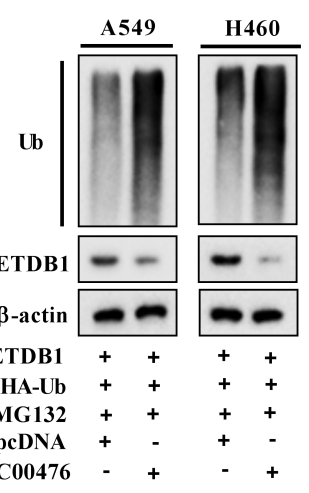

D

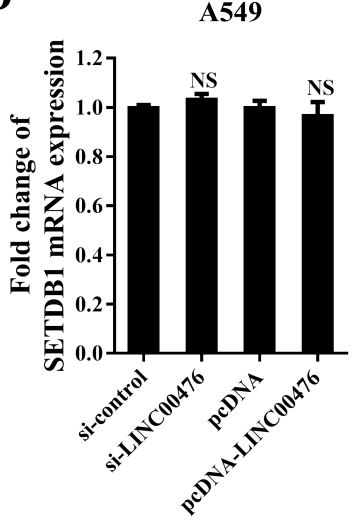

H460

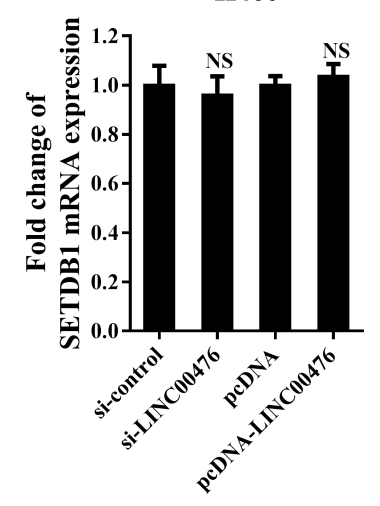

$\mathbf{E}$
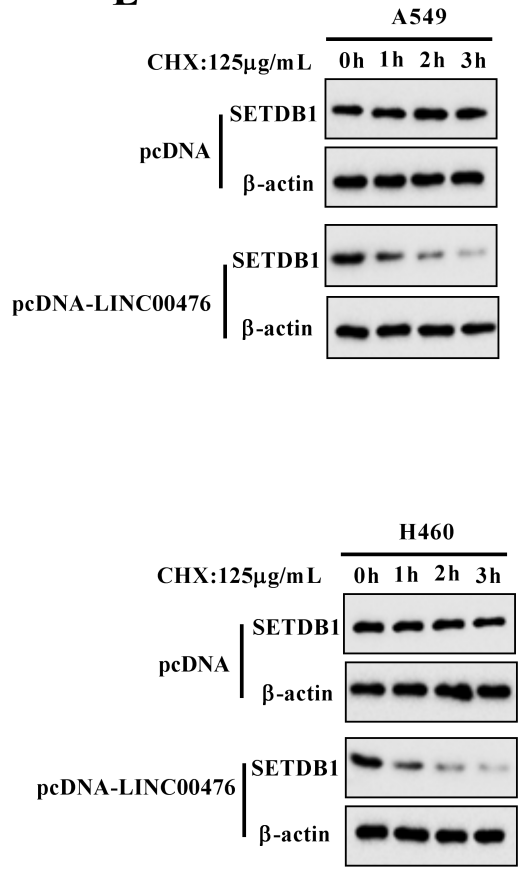

A549

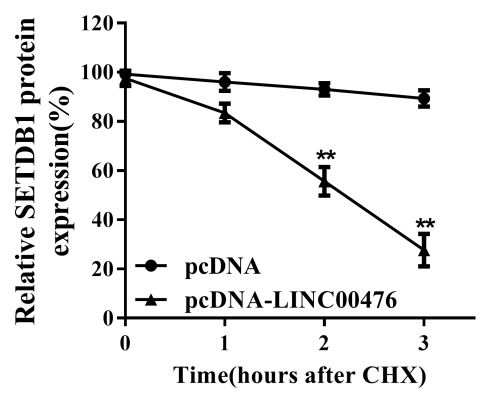

H460

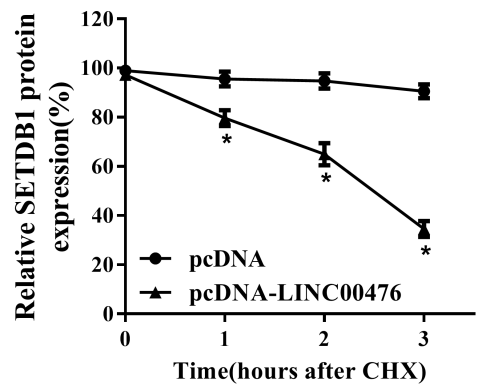

Figure 3

LINC00476 induces the degradation of SETDB1 via promoting its ubiquitination A. RNA pull-down assay showed that SETDB1 was expressed in the LINC00476 pull-down compound. B. RNA immunoprecipitation (RIP) assay showed that the fold change of LINC00476 was increased in SETDB1immunoprecipitated complex compared with the lgG-immunoprecipitated complex. A549 and H460 cells were transfected with the LINC00476 overexpression plasmid (pcDNA-LINC00476) or the control plasmid (pcDNA), or transfected with the LINC00476 small interfering RNA (si-LINC00476) or the control siRNA (sicontrol) for $48 \mathrm{~h}$. The protein expression (C) and the mRNA expression (D) of SETDB1 were determined using western blot analysis and qRT-PCR, respectively. E. The protein level of SETDB1 was determined at 1,2 , and $3 \mathrm{~h}$ after the treatment of the protein synthesis inhibitor $\mathrm{CHX}(125 \mu \mathrm{g} / \mathrm{mL})$. F. A549 and $\mathrm{H} 460$ cells were transfected with HA-Ub, Flag-SETDB1, and pcDNA/pcDNA-LINC00476 for $48 \mathrm{~h}$. The proteasome inhibitor MG132 was added for $4 \mathrm{~h}$ before cell collection. Cell lysates were 
immunoprecipitated with anti-SETDB1 and were immunoblotted with anti-HA. ${ }^{*} p<0.05,{ }^{* *} p<0.01$ vs pcDNA.

A

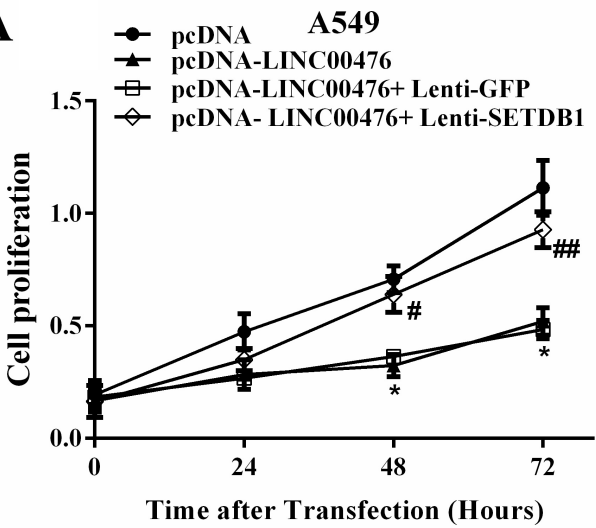

B

A549

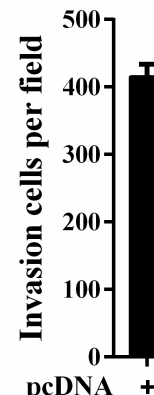

pcDNA +

pcDNA-LINC00476

Lenti-GFP

Lenti-SETDB1

C

A549

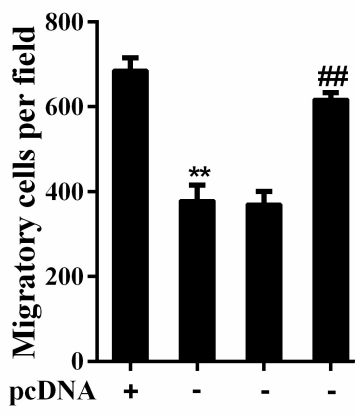

pcDNA-LINC00476 - + + + +

Lenti-GFP

Lenti-SETDB1

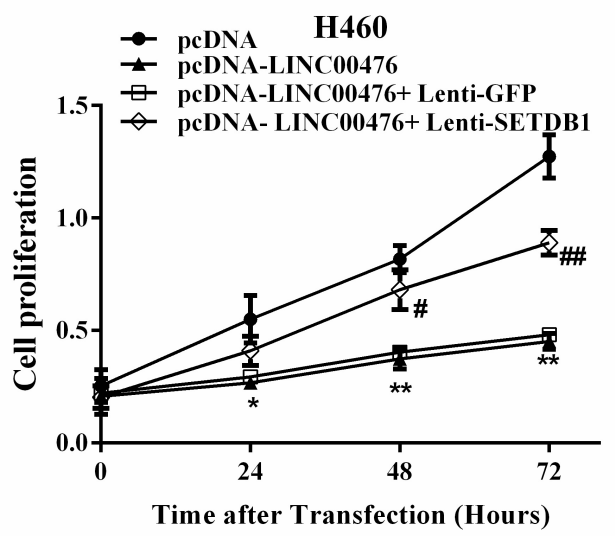

H460

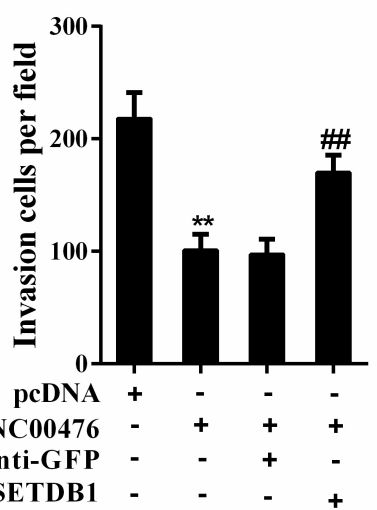

D

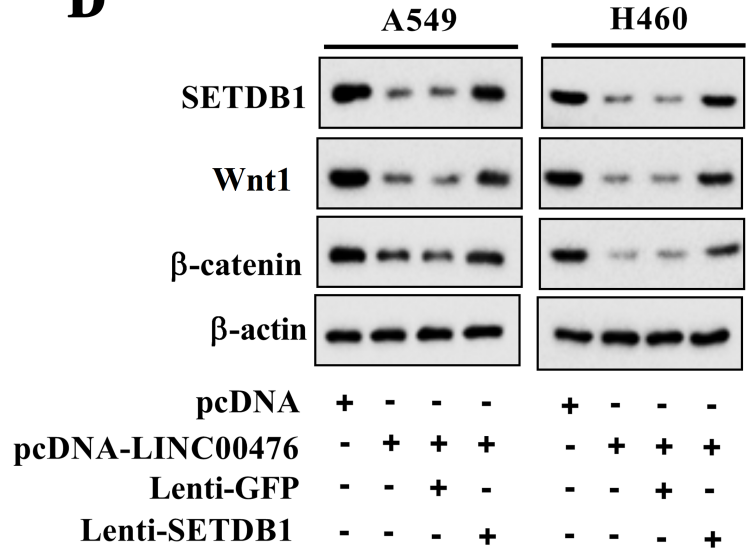

H460

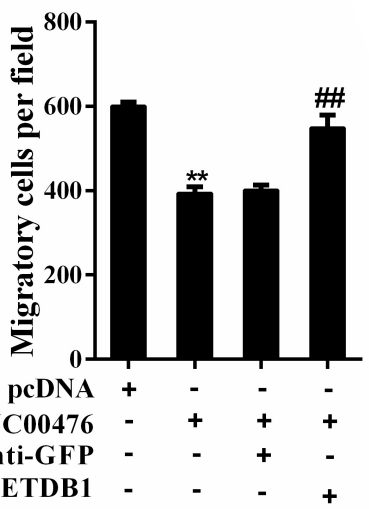

\section{Figure 4}

LINC00476 suppresses NSCLC cell proliferation, invasion, and migration via the SETDB1/Wnt/ $\beta$-catenin axis A549 and H460 cells were transfected with LINC00476 overexpression plasmid (pcDNA-LINC00476), or co-transfected with SETDB1 overexpression lentivirus (Lenti-SETDB1) for $48 \mathrm{~h}$. The cell proliferation $(A)$, the cell invasion $(B)$ and the cell migration $(C)$ were determined using cell counting kit-8 (CCK-8) assay and Transwell assay. D. The protein expressions of SETDB1, Wnt1, and $\beta$-catenin were determined using western blot analysis. 